\title{
Event recorder monitoring to compare the efficacy of a left versus biatrial lesion set in patients undergoing concomitant surgical ablation for atrial fibrillation
}

\author{
Simon Pecha, MD, ${ }^{\mathrm{a}}$ Friederike Hartel, MD, ${ }^{\mathrm{b}}$ Teymour Ahmadzade, MD, ${ }^{\mathrm{a}}$ Muhammet Ali Aydin, MD, \\ Stephan Willems, MD, ${ }^{\mathrm{b}}$ Hermann Reichenspurner, $\mathrm{MD}, \mathrm{PhD},{ }^{\mathrm{a}}$ and Florian Mathias Wagner, $\mathrm{MD}^{\mathrm{a}}$
}

\begin{abstract}
Objectives: Various lesion sets and subsequent success rates have been reported in patients receiving concomitant surgical ablation for atrial fibrillation. However, most of these results have been obtained by discontinuous monitoring. We report results using continuous event recorder rhythm monitoring to compare more accurately the efficacy of a left versus biatrial lesion set to treat patients with persistent atrial fibrillation.
\end{abstract}

\begin{abstract}
Methods: Between July 2008 and December 2011, 66 patients with persistent or long-standing persistent atrial fibrillation underwent concomitant surgical atrial fibrillation ablation with a biatrial lesion set and subcutaneous event recorder implantation. The results and outcomes were compared with a propensity score-matched cohort of 66 patients with a left atrial lesion set and event recorder implantation. Event recorder interrogation was performed at 3, 6, and 12 months follow-up.
\end{abstract}

\begin{abstract}
Results: The mean patient age was $70.2 \pm 7.4$ years, and $70.3 \%$ were male. No major ablation-related complications occurred. One-year survival was $94.8 \%$ with no statistically significant differences between the 2 groups. The overall rate of freedom from atrial fibrillation was $57.3 \%$ and $64.4 \%$ after 3 and 12 months follow-up, respectively. Three months postoperatively, patients in the biatrial group had a slightly higher rate of freedom from atrial fibrillation $(63.6 \%$ vs $52.3 \% P=.22)$, but it did not reach statistical significance. At 12 months follow-up, a statistically significant higher rate of freedom from atrial fibrillation was observed in patients with a biatrial lesion set $(74.4 \%$ vs $55.8 \% ; P=.026)$. The mean atrial fibrillation burden in all patients was $15.1 \% \pm 12.5 \%$ in the biatrial group and $21.2 \% \pm 14.4 \%$ in the left atrial group 12 months postoperatively $(P=.03)$.
\end{abstract}

Conclusions: Continuous rhythm monitoring by subcutaneous event recorder implantation was safe and feasible. In patients undergoing biatrial ablation, a statistically significant higher rate of freedom from atrial fibrillation was observed at 12 months follow-up. (J Thorac Cardiovasc Surg 2014;148:2161-6)

Atrial fibrillation (AF) is associated with an increased number of thromboembolic events, including stroke. Furthermore, it can lead to heart failure and results in an increased number of hospitalizations. ${ }^{1,2}$ Therefore, concomitant surgical AF ablation is recommended by guidelines for symptomatic patients and for asymptomatic patients at low risk for the surgical ablation procedure. ${ }^{2,3}$ Cox first reported his surgical AF ablation technique using the cut-and-sew principle in 1987, which was subsequently revised to the Cox-Maze III procedure.

\footnotetext{
From the Departments of Cardiovascular Surgery ${ }^{\mathrm{a}}$ and Cardiology/Electrophysiology, ${ }^{\mathrm{b}}$ University Heart Center Hamburg, Hamburg, Germany.

Disclosures: Stephan Willems reports consulting fees from St. Jude Medical and Boehringer Ingelheim and lecture fees from Bayer. Muhammet Ali Aydin reports lecture fees from Medtronic, St. Jude Medical, and Biotronik. All other authors have nothing to disclose with regard to commercial support.

Received for publication Nov 11, 2013; revisions received Feb 12, 2014; accepted for publication Feb 21, 2014; available ahead of print March 22, 2014

Address for reprints: Simon Pecha, MD, Department of Cardiovascular Surgery, University Heart Center Hamburg, Martinistr. 52, 20246 Hamburg, Germany (E-mail: s.pecha@uke.de).

0022-5223/\$36.00

Copyright (c) 2014 by The American Association for Thoracic Surgery http://dx.doi.org/10.1016/j.jtcvs.2014.02.061
}

Because of success rates greater than $90 \%$, the Cox-Maze III procedure became the gold standard for surgical AF ablation. However, because of the complexity of the procedure, it was performed by only a few surgeons. After the cut-and-sew principle was recently replaced with the creation of transmural thermal lesions via the application of different energy sources, such as radiofrequency, high-frequency ultrasound, or cryotherapy, the use of the procedure has become widespread. This procedure is known as the "Cox-Maze IV" and contains a similar lesion set as in the initial Cox-Maze III procedure, including lesions in both atria. ${ }^{4}$

Different modifications and simplifications of the original lesion set have been used over the years. In most patients, AF originates at the pulmonary veins, especially in patients with paroxysmal AF, and in such cases, a left atrial lesion set is considered sufficient by many specialists. However, in patients with persistent or long-standing persistent AF, there is an ongoing discussion about whether a biatrial lesion set results in higher success rates. ${ }^{4-8}$ All of the recent published studies comparing the efficacy of different lesion sets used discontinuous rhythm monitoring. However, studies comparing the efficacy of different follow-up 


\section{Abbreviations and Acronyms \\ $\mathrm{AF}=$ atrial fibrillation \\ $\mathrm{ECG}=$ electrocardiography \\ $\mathrm{ER}=$ event recorder}

methods after surgical AF ablation have shown that short time rhythm monitoring, even with repeated 24-hour Holter electrocardiography (ECG), underestimates the rate of AF recurrence. ${ }^{9}$ The Reveal XT Performance Trial (XPECT) trial showed high sensitivity $(96.1 \%)$ and negative predictive value $(97.4 \%)$ for the detection of AF episodes by the Reveal XT (Medtronic Inc, Minneapolis, Minn) device, ${ }^{10}$ a subcutaneous implantable event recorder (ER). This device is programmed to detect arrhythmia episodes by analysis of irregularity and incoherence of R-R intervals. The Reveal $\mathrm{XT}$ is able to detect the duration of AF episodes and burden, defined as the percentage of time the patient is in AF during follow-up. The aim of our study was to compare the efficacy of left and biatrial lesion sets through continuous rhythm monitoring using subcutaneous ER implantation.

\section{MATERIALS AND METHODS}

From July 2008 to December 2011, 255 patients underwent concomitant surgical ablation because of persistent or long-standing persistent AF. Of those patients, 88 received biatrial ablation and 167 received left atrial ablation. Classification of persistent and long-standing persistent AF was according to Heart Rhythm Society/European Heart Rhythm Association/European Cardiac Arrhythmia Society expert consensus statement on catheter and surgical ablation of $\mathrm{AF}^{3}$ Propensity score matching resulted in a group of 66 patients who received a biatrial lesion set and subcutaneous ER (Reveal XT) implantation and a control group of 66 patients who received a left atrial lesion set and ER implantation. All patients received implantation of the Reveal device during surgical ablation procedure. Patients were matched by age, gender, left ventricular ejection fraction, left atrial diameter, AF duration, surgical procedure, and energy source type.

In group 1, biatrial ablation was performed in 66 patients, including left atrial bilateral pulmonary vein ablation, box lesion, and left atrial appendage and isthmus isolation. Right sided-lesion set included an intercaval lesion and isolation of the cavotricuspid isthmus, right atrial appendage, and terminal crest. In group 2, only the left atrial lesion set was conducted. Applied energy sources included argon-based cryoablation (cryoICE cryoablation probe, AtriCure Inc, West Chester, Ohio; Cardioblate CryoFlex Surgical Ablation Probe, Medtronic Inc) in 21 patients (biatrial, $\mathrm{n}=12$; left atrial, $\mathrm{n}=9 ; P=.63$ ), unipolar radiofrequency ablation (Cardioblate unipolar RF pen, Medtronic Inc) in 52 patients (biatrial, $\mathrm{n}=28$; left atrial, $\mathrm{n}=24 ; P=.37$ ), and bipolar ablation (Cardioblate BP2 device and Cardioblate Surgical Ablation System Generator, Medtronic Inc; Atricure Isolator Synergy Ablation Clamp, AtriCure Inc) in 59 patients (biatrial, $\mathrm{n}=26$; left atrial, $\mathrm{n}=33$ ). None of these energy sources were used in combination.

\section{Statistical Analysis}

All statistical analyses were performed using SPSS version 18.0 (SPSS Inc, Chicago, Ill). Continuous values are expressed as mean \pm standard deviation and were compared using the Student $t$ test or Mann-Whitney test as appropriate. Categoric variables are displayed as frequencies, and percentages were compared using the chi-square test or Fisher exact test as appropriate. Reported $P$ values are 2-sided. A logistic regression model was used to generate a control group with a left atrial lesion set matched for the variables detailed earlier. Matching was performed by selecting a patient randomly from the biatrial group and identifying a partner in the control group with the nearest logit-transformed propensity score. Matching balance was assessed using statistical analysis with the Fisher exact test for categoric variables and the Student $t$ test for continuous variables.

\section{Follow-up}

Follow-up with ER interrogation was conducted 3, 6, and 12 months postoperatively. AF recurrence was defined as an $\mathrm{AF}$ burden greater than $0.5 \%$ or a single stored AF episode with duration more than 30 seconds on ER interrogation. However, one needs to realize that the Reveal ER has a blanking period of 2 minutes because R-R intervals are analyzed within each 2-minute period of time. When there is a uncorrelated irregularity of the R-R intervals within the 2-minute interval, the heart rhythm in this period is classified as AF. All stored episodes were manually validated during follow-up visits. The primary end point of the study was freedom from AF after 12 months. To obtain success rate at 12 months, stored $\mathrm{AF}$ episodes from ER interrogation between 6 and 12 months were included. The postoperative and discharge rhythm results were obtained using 12-lead ECG. The antiarrhythmic drugs and anticoagulation regimens were maintained for 3 months postoperatively in all patients and then adapted according to the ER rhythm results. In patients without contraindications, amiodarone was used as the first-line antiarrhythmic drug therapy; otherwise, other class I or III antiarrhythmic drugs were used for at least 3 months postoperatively.

\section{RESULTS}

Baseline patient characteristics are shown in Table 1. Because of the matching process, there were no statistically significant differences between the 2 groups. The mean patient age was $70.1 \pm 7.5$ years in the left atrial group and $70.5 \pm 7.3$ years in the biatrial group. The mean left ventricular ejection fraction was $51.5 \% \pm 10.9 \%$ in patients with a left atrial lesion set and $51.9 \% \pm 10.8 \%$ in the biatrial group. The mean left atrial diameter was $53.6 \pm 7.5 \mathrm{~mm}$ for patients with a left atrial lesion set and $54.9 \pm 8.0 \mathrm{~mm}$ in patients with biatrial ablation. The mean AF duration was $3.9 \pm 3.2$ years in patients with a left atrial set and $4.5 \pm 3.8$ years for patients with a biatrial lesion set. Surgical procedures and applied energy sources were equally distributed between the 2 groups (Table 2).

No major ablation or ER-associated complications occurred in any of the patients. There were no cases of intraoperative death. The in-hospital mortality rate was $2.2 \%$ (3/132) (biatrial, $3.0 \% 2 / 66$; left atrial, $1.5 \% 1 / 66$; $P=.25)$, whereas the 30 -day mortality rate was $3.0 \%$ (4/132) (biatrial, 3.0\%; left atrial, 3.0\%). The 1-year survival was $94.8 \%(125 / 132)$, and there were no statistically significant differences between the 2 groups (biatrial, $95.6 \%$; left atrial, $93.9 \% P=.68$ ). Three of 125 patients $(2.4 \%)$ experienced perioperative stroke (left atrial, 2/63 [1.8\%]; biatrial, $\mathrm{n}=1 / 62[1.0 \%])$. Postoperative new permanent pacemaker implantation rate was $11.2 \%(7 / 62)$ and $6.5 \%(4 / 63)(P=.5)$ in the biatrial and left atrial groups, respectively. 
TABLE 1. Patient demographics

\begin{tabular}{lccc}
\hline & $\begin{array}{c}\text { Left atrial } \\
\mathbf{n}=\mathbf{6 6}\end{array}$ & $\begin{array}{c}\text { Biatrial } \\
\mathbf{n = 6 6}\end{array}$ & $\boldsymbol{P}$ value \\
\hline Age (y) & $70.1 \pm 7.5$ & $70.5 \pm 7.3$ & .64 \\
Gender (male n, \%) & $40(60.1)$ & $45(68.2)$ & .46 \\
AF duration (y) & $3.9 \pm 3.2$ & $4.5 \pm 3.8$ & .33 \\
LA diameter (mm) & $53.6 \pm 7.5$ & $54.9 \pm 8.0$ & .27 \\
Long-standing persistent AF, n (\%) & $54(81.8)$ & $58(87.9)$ & .46 \\
Persistent AF, n (\%) & $12(18.1)$ & $8(12.1)$ & .47 \\
LVEF (\%) & $51.5 \pm 10.9$ & $51.9 \pm 10.8$ & .81 \\
Diabetes, n (\%) & $13(19.7)$ & $16(24.2)$ & .67 \\
Renal insufficiency, n (\%) & $7(10.6)$ & $5(7.6)$ & .76 \\
Prior stroke, n (\%) & $5(7.6)$ & $6(9.1)$ & .77 \\
COPD, n (\%) & $8(12.1)$ & $7(10.6)$ & 1.0 \\
CAD, n (\%) & $34(51.5)$ & $39(59.1)$ & .48 \\
\hline
\end{tabular}

$A F$, Atrial fibrillation; $C A D$, coronary artery disease; $C O P D$, chronic obstructive pulmonary disease; $L A$, left atrial; $L V E F$, left ventricular ejection fraction.

\section{Rhythm Results}

All patients underwent ER interrogation at 3 and 12 months of follow-up. Six-month follow-up was performed in $61.7 \%$ of patients $(69 / 132)$. There was no statistically significant difference between the 2 groups in terms of freedom from AF immediately after the procedure and at the time of discharge (left atrial $51.4 \%$ [34/66] vs $62.1 \%$ [41/66], $P=.28$ and left atrial $51.1 \%$ [33/65] vs $60.6 \%$ [41/64], $P=.28$, respectively) (Figure 1). At 3 and 6 months of follow-up, there were no significant differences in freedom from AF between the left atrial and biatrial groups (left atrial $53.2 \%$ [33/62] vs $63.9 \%$ [39/ 61 ], $P=.22$ and $56.3 \%$ [18/32] vs $67.3 \%$ [25/37], $P=.20$, respectively). However, it must be considered that only $61.7 \%$ of the patients underwent a 6-month follow-up.

One-year follow-up revealed a significantly different rate of freedom from AF of 55.8\% (35/63) for patients with left atrial ablation compared with $74.4 \%(46 / 62)$ for patients with a biatrial lesion set $(P=.026)$ (Figure 1). The rate of freedom from $\mathrm{AF}$ at 12 months follow-up off class I

TABLE 2. Surgical procedures and ablation energy sources

\begin{tabular}{lccc}
\hline & Left atrial $\mathbf{n}=\mathbf{6 6}$ & Biatrial $\mathbf{n}=\mathbf{6 6}$ & $\boldsymbol{P}$ value \\
\hline CABG, n (\%) & $21(31.8)$ & $17(25.8)$ & .56 \\
AVR, n (\%) & $10(15.2)$ & $7(10.6)$ & .60 \\
MVR, n (\%) & $16(24.2)$ & $22(33.3)$ & .34 \\
CABG + valve & $11(16.7)$ & $13(19.7)$ & .82 \\
MVR + TVR, n (\%) & $4(6.1)$ & $2(3.0)$ & .67 \\
Other, n (\%) & $4(6.1)$ & $5(7.6)$ & 1.0 \\
RF bipolar, n (\%) & $33(50.0)$ & $26(39.4)$ & .16 \\
RF unipolar, n (\%) & $24(36.4)$ & $28(42.4)$ & .37 \\
Cryoablation, n (\%) & $9(13.6)$ & $12(18.2)$ & .63 \\
\hline
\end{tabular}

$A V R$, Aortic valve replacement; $C A B G$, coronary artery bypass grafting; $M V R$, mitral valve repair or replacement; $R F$, radiofrequency; $T V R$, tricuspid valve repair.

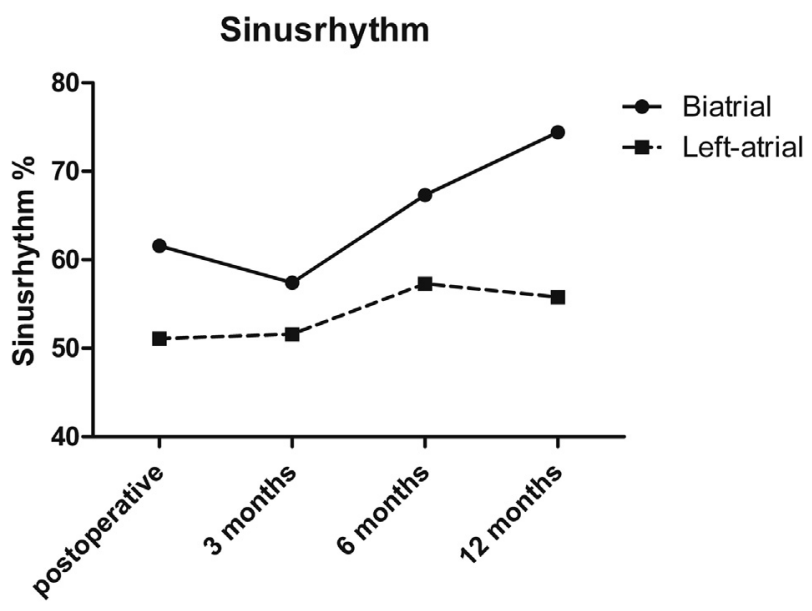

FIGURE 1. Sinus rhythm rates during follow-up.

and III antiarrhythmic drugs was 50.7\% (32/63) and $68.1 \%(42 / 66)$, respectively $(P=.033)$. However, freedom from oral anticoagulation at 12 months follow-up was only $25.4 \%(16 / 63)$ in the biatrial group and $24.1 \%(15 / 62)$ in the left atrial group.

\section{Atrial Fibrillation Burden}

The mean AF burden of all patients was $25 \%$ in the biatrial group and $39 \%$ in the left atrial group at 3 months of follow-up. At 12 months of follow-up, a reduced AF burden was seen in all patients irrespective of ablation success. The AF burden in the entire cohort was $15.1 \%$ $\pm 12.5 \%(\mathrm{n}=62)$ in the biatrial group and $21.3 \% \pm$ $14.4 \%(\mathrm{n}=63)$ in the left atrial group 12 months postoperatively $(P=.03)$. For patients in whom ablation failed, the mean AF burdens of $35 \%(n=62)$ and $43 \%(n=63)$ were seen in the biatrial and left atrial groups, respectively.

\section{DISCUSSION}

In the literature, no consensus has been reached about the appropriate lesion set for patients with different types of AF. Specifically, there is a controversial discussion on which patients should be treated with an additional right-sided lesion set. In our study, we showed a statistically higher rate of sinus rhythm after 12 months in patients with persistent or long-standing persistent $\mathrm{AF}$ treated with a biatrial lesion set. This finding is consistent with a previous published meta-analysis by Barnett and $\mathrm{Ad}^{4}$ that included 69 studies with an overall number of 5885 patients with AF who received cardiac surgery alone or cardiac surgery with concomitant surgical ablation. In their analysis, the patients who received surgical ablation had statistically significant higher rates of sinus rhythm at all points of follow-up to 3 years. Furthermore, patients who had received the biatrial lesion set had statistically 
higher rates of freedom from AF than those who received the left atrial lesion set. Similar results were shown in a meta-analysis by Cheng and colleagues ${ }^{11}$ that included 4647 patients with persistent or long-standing persistent AF. However, a higher incidence of postoperative permanent pacemaker dependency for patients receiving biatrial ablation has been shown in previous published studies, and caution is recommended while performing right-sided lesions. . $^{12,13}$

In contrast to the results published by Barnett and $\mathrm{Ad}^{4}$ and Cheng and colleagues, ${ }^{11}$ other studies have indicated no difference in rhythm results between patients who received left atrial and biatrial ablation. A recent study by Soni and colleagues ${ }^{14}$ showed the rates of freedom from $\mathrm{AF}$ in patients who underwent biatrial lesion set, left atrial lesion set, and pulmonary vein isolation were $80.0 \%, 76.1 \%$, and $56.9 \%$, respectively, at 12 months of follow-up. This analysis suggested no improvement in patients who underwent an additional right atrial lesion set. ${ }^{14}$ The limitation of this study is that the success rates were obtained using 12-lead ECG, but a more extensive follow-up with at least a 24-hour Holter ECG would probably show different results.

However, it needs to be stressed that in most of the previous published studies, ablation success was defined by use of 12-lead ECG or 24-hour Holter monitoring. ${ }^{4-6,12,15-17}$ Recent studies comparing the accuracy of rhythm detection using different follow-up methods after AF ablation showed that intermittent rhythm monitoring underestimates the actual AF recurrence rate. ${ }^{9,17}$ Furthermore, after $\mathrm{AF}$ ablation, even patients in whom ablation failed may have reduced $\mathrm{AF}$ burden, which makes the detection of $\mathrm{AF}$ recurrence with snapshot follow-up methods more difficult and unreliable. Our study was the first to assess success rates of biatrial and left atrial lesion sets using continuous subcutaneous implanted ER monitoring. We demonstrated superior results concerning freedom from AF recurrence in patients receiving biatrial ablation after 1 year of follow-up. The use of continuous rhythm monitoring in our study allows for a more accurate comparison of the 2 different lesion sets, because the sensitivity to detect recurrent AF episodes is high with this method. However, the ER used in this study has a limited storage capacity of 49.5 minutes. Therefore, in patients with a high AF burden, not all stored episodes can be manually validated, and there is a certain risk to over- or underestimate the AF burden. Furthermore, the Reveal ER classifies the heart rhythm for each subsequent time interval of 2 minutes and can only detect $\mathrm{AF}$ episodes with a duration of at least 2 minutes. This means that every stored episode in the Reveal ER corresponds to an AF duration of more than 2 minutes and represents a failure of ablation according to the guidelines. Despite this 2-minute blanking period, in previous published studies it has been shown that the Reveal device is an excellent tool to predict freedom from $\mathrm{AF}$ because of its high negative predictive values. ${ }^{10}$ Despite these limitations, the ability to detect AF episodes is far better than with 24-hour, 7day, or 30-day monitoring,,$^{9,18,19}$ and in our opinion, ER monitoring is the best available monitoring strategy to date.

An important observation in our study was the increase in the sinus rhythm rate between 3 and 12 months followup. The current study also showed a reduction of $\mathrm{AF}$ burden over time, even in patients in whom ablation failed. However, the impact of reduction in AF burden and the resulting consequences remain undefined yet. Future larger, prospective studies need to be performed to determine clinically relevant cutoff values for the AF burden in regard to the anticoagulation and antiarrhythmic drug regimens.

Our study did not show a survival benefit for patients with restored sinus rhythm. Furthermore, no significant differences were observed in the survivals of patients with a left or biatrial lesion set. These results may be due to the relatively short 12-month follow-up period and the limited number of included patients. Regardless, this finding is consistent with the results of the meta-analysis by Barnett and colleagues, ${ }^{4}$ in which similar survivals were reported for patients with and without successful ablation. In contrast, in a propensity score-matched analysis by Lee and colleagues, ${ }^{20}$ patients with AF receiving surgical ablation had similar survival compared with patients without a history of AF. Furthermore, they showed a survival benefit for patients with successful ablation compared with those in whom AF ablation failed. ${ }^{20}$ These first promising results have to be confirmed in larger, prospective, randomized trials to determine the impact of AF surgery on long-term survival.

After 1 year of follow-up, no statistically significant difference was observed between the left and biatrial ablation groups in regard to stroke-free survival in the current study. This finding may be the result of low incidences of postoperative stroke in both groups, which may be one of the advantages of left atrial appendage closure or excision, which can be easily done during surgical $\mathrm{AF}$ ablation.

Measurement of AF burden is a relatively new method to determine the time a patient spent in AF. If a patient has an $\mathrm{AF}$ burden of $0 \%$ over a certain period of time, we consider the ablation as successful and the patient as $\mathrm{AF}$ free. Nevertheless, the impact of reduction in AF burden and the resulting consequences remain undefined. Future larger prospective studies need to be performed to determine clinically relevant cutoff values for the AF burden 
in regard to the anticoagulation and antiarrhythmic drug regimen.

\section{Study Limitations}

The major limitation of this study is that we used a retrospective data analysis to compare the biatrial and left atrial lesion set groups. Although we minimized the differences between the 2 groups by matching the patients using a logistic regression model, the disadvantages of a retrospective, nonrandomized study, including unknown confounders and selection and detection bias, cannot be completely avoided.

Further limitations are due to technical aspects of ER with its limited storage capacity of 49.5 minutes. Therefore, in patients with a high AF burden, not all stored episodes can be validated manually, and there is a certain risk to over- or underestimate AF burden. Furthermore, the ER used in this study classifies the heart rhythm for each subsequent time interval of 2 minutes and only detects $\mathrm{AF}$ episodes with a duration of at least 2 minutes. This 2-minute blanking period of the ER may result in underestimation of AF episodes. Additional limitations of the study are that we did not use intraoperative electrophysiologic mapping and testing to confirm the quality of the applied lesions, and that no follow-up beyond 12 months is available.

We started to perform AF surgery in 2002 and initially used only left atrial lesions in all patients. As a result of the published data and our own experience, in 2008 we introduced the use of biatrial lesions in patients with persistent and long-standing persistent AF. Therefore, the results of the biatrial ablation may be influenced by a certain learning curve.

Moreover, the heterogeneity of the energy sources used is a limitation of the study. Furthermore, there was no consistent treatment of left atrial appendage (excision or to exclusion), and we did not routinely use transesophageal echocardiography postoperatively to control the success of left atrial appendage management.

\section{CONCLUSIONS}

In the current study, in which all rhythm results were obtained by ER monitoring, we showed that a biatrial lesion set in patients with persistent AF results in statistically significant higher sinus rhythm rates at 12 months follow-up. Furthermore, over time, patients showed a reduction of AF burden, even those in whom ablation failed.

\section{References}

1. Flaker GC, Belew K, Beckman K, et al. Asymptomatic atrial fibrillation: demographic features and prognostic information from the Atrial Fibrillation Followup Investigation of Rhythm Management (AFFIRM) study. Am Heart J. 2005; 149:657-63.
2. Fuster V, Ryden LE, Cannom DS, et al. 2011 ACCF/AHA/HRS focused updates incorporated into the ACC/AHA/ESC 2006 Guidelines for the management of patients with atrial fibrillation: a report of the American College of Cardiology Foundation/American Heart Association Task Force on Practice Guidelines developed in partnership with the European Society of Cardiology and in collaboration with the European Heart Rhythm Association and the Heart Rhythm Society. J Am Coll Cardiol. 2011;57: e101-98.

3. Calkins H, Kuck KH, Cappato R, et al. 2012 HRS/EHRA/ECAS expert consensus statement on catheter and surgical ablation of atrial fibrillation: recommendations for patient selection, procedural techniques, patient management and follow-up, definitions, endpoints, and research trial design: a report of the Heart Rhythm Society (HRS) Task Force on Catheter and Surgical Ablation of Atrial Fibrillation. Developed in partnership with the European Heart Rhythm Association (EHRA), a registered branch of the European Society of Cardiology (ESC) and the European Cardiac Arrhythmia Society (ECAS); and in collaboration with the American College of Cardiology (ACC), American Heart Association (AHA), the Asia Pacific Heart Rhythm Society (APHRS), and the Society of Thoracic Surgeons (STS). Endorsed by the governing bodies of the American College of Cardiology Foundation, the American Heart Association, the European Cardiac Arrhythmia Society, the European Heart Rhythm Association, the Society of Thoracic Surgeons, the Asia Pacific Heart Rhythm Society, and the Heart Rhythm Society. Heart Rhythm. 2012;9:632-96.e21.

4. Barnett SD, Ad N. Surgical ablation as treatment for the elimination of atrial fibrillation: a meta-analysis. J Thorac Cardiovasc Surg. 2006;131: 1029-35.

5. Abreu Filho CA, Lisboa LA, Dallan LA, et al. Effectiveness of the maze procedure using cooled-tip radiofrequency ablation in patients with permanent atrial fibrillation and rheumatic mitral valve disease. Circulation. 2005; 112(9 Suppl):I20-5.

6. Schuetz A, Schulze CJ, Sarvanakis KK, et al. Surgical treatment of permanent atrial fibrillation using microwave energy ablation: a prospective randomized clinical trial. Eur J Cardiothoracic Surg. 2003;24:475-80.

7. Gillinov AM, Bakaeen F, McCarthy PM, et al. Surgery for paroxysmal atrial fibrillation in the setting of mitral valve disease: a role for pulmonary vein isolation? Ann Thorac Surg. 2006;81:19-28.

8. Gillinov AM, Bhavani S, Blackstone EH, et al. Surgery for permanent atrial fibrillation: impact of patient factors and lesion set. Ann Thorac Surg. 2006; 82:502-14.

9. Hanke T, Charitos EI, Stierle U, et al. Twenty-four-hour Holter monitor followup does not provide accurate heart rhythm status after surgical atrial fibrillation ablation therapy: up to 12 months experience with a novel permanently implantable heart rhythm monitor device. Circulation. 2009;120(11 Suppl): S177-84.

10. Hindricks G, Pokushalov E, Urban L, et al. Performance of a new leadless implantable cardiac monitor in detecting and quantifying atrial fibrillation: results of the XPECT trial. Circ Arrhythm Electrophysiol. 2010;3: $141-7$.

11. Cheng DC, Ad N, Martin J, et al. Surgical ablation for atrial fibrillation in cardiac surgery: a meta-analysis and systematic review. Innovations. 2010;5: 84-96.

12. Worku B, Pak SW, Cheema F, et al. Incidence and predictors of pacemaker placement after surgical ablation for atrial fibrillation. Ann Thorac Surg. 2011;92: 2085-9.

13. Pecha S, Schafer T, Yildirim Y, et al. Predictors for permanent pacemaker implantation after concomitant surgical ablation for atrial fibrillation. J Thorac Cardiovasc Surg. 2014;147:984-8.

14. Soni LK, Cedola SR, Cogan J, et al. Right atrial lesions do not improve the efficacy of a complete left atrial lesion set in the surgical treatment of atrial fibrillation, but they do increase procedural morbidity. J Thorac Cardiovasc Surg. 2013; 145:356-63.

15. Deneke T, Khargi K, Grewe PH, et al. Efficacy of an additional MAZE procedure using cooled-tip radiofrequency ablation in patients with chronic atrial fibrillation and mitral valve disease. A randomized, prospective trial. Eur Heart J. 2002;23:558-66.

16. Doukas G, Samani NJ, Alexiou C, et al. Left atrial radiofrequency ablation during mitral valve surgery for continuous atrial fibrillation: a randomized controlled trial. JAMA. 2005;294:2323-9. 
17. Blomstrom-Lundqvist C, Johansson B, Berglin E, et al. A randomized double-blind study of epicardial left atrial cryoablation for permanent atrial fibrillation in patients undergoing mitral valve surgery: the SWEDish Multicentre Atrial Fibrillation study (SWEDMAF). Eur Heart J. 2007;28: 2902-8.

18. Ziegler PD, Koehler JL, Mehra R. Comparison of continuous versus intermittent monitoring of atrial arrhythmias. Heart Rhythm. 2006;3: $1445-52$.
19. Dagres N, Kottkamp H, Piorkowski C, et al. Influence of the duration of Holter monitoring on the detection of arrhythmia recurrences after catheter ablation of atrial fibrillation: implications for patient follow-up. Int J Cardiol. 2010;139: 305-6.

20. Lee R, McCarthy PM, Wang EC, et al. Midterm survival in patients treated for atrial fibrillation: a propensity-matched comparison to patients without a history of atrial fibrillation. J Thorac Cardiovasc Surg. 2012; $143: 1341-51$. 